\title{
Connecting the NOAA National Marine Sanctuaries through Telepresence
}

Michiko Martin

NOAA-National Marine Sanctuaries • Silver Spring, Maryland USA

The National Oceanic and Atmospheric Administration's (NOAA) National Marine Sanctuary (NMS) Program has established an innovative educational and technological framework to bring the excitement of the nation's underwater ecosystems to the American public by linking telepresence activities with system-wide oceanographic monitoring programs. This initiative will revolutionize oceanographic information dissemination by integrating live video camera feeds with data streams as diverse as observations collected by citizen-scientists, satellite records, and measurements collected by monitoring stations in the marine sanctuaries. Integration is achieved through distributed data systems and protocols that enable unified internet access to information collected at multiple sites through multiple observing systems.

Developed in partnership with the JASON Foundation for Education, the Institute for Exploration, and MOTE Marine Lab, NOAA's framework allows individuals living far from the coastal zone to experience up-close the wonders of these special marine areas without even getting their feet wet. A successful telepresence pilot began operating in Monterey Bay National Marine Sanctuary in 2002, allowing visitors to the Mystic (Connecticut) Aquarium's "Immersion Theater" to view and operate, in real time, a remotely operated vehicle tethered near a kelp forest in 50 feet of water. The popular exhibit has introduced thousands of visitors to the underwater wonders of the Monterey Bay ecosystem.

NOAA's National Marine Sanctuary Program is partnering with aquaria, research institutions, and a variety of private and federal partners to expand this exciting technology to the Channel Islands, Florida Keys and Thunder Bay sanctuaries over the next two years. The vision is to eventually establish a telepresence and monitoring capability at all thirteen national marine sanctuaries and to provide the public the ability to analyze the movement of satellite-tagged loggerhead turtles in the South Atlantic Bight, observe sea otters in the Monterey Bay kelp forest, listen to the songs of the Hawaiian Islands humpback whales, and interact with this information in its broader context to address current environmental and social challenges. By linking telepresence with system-wide monitoring, this initiative promises to excite and educate millions by bringing the National Marine Sanctuaries and their vibrant ecosystems right into the homes of audience members worldwide.
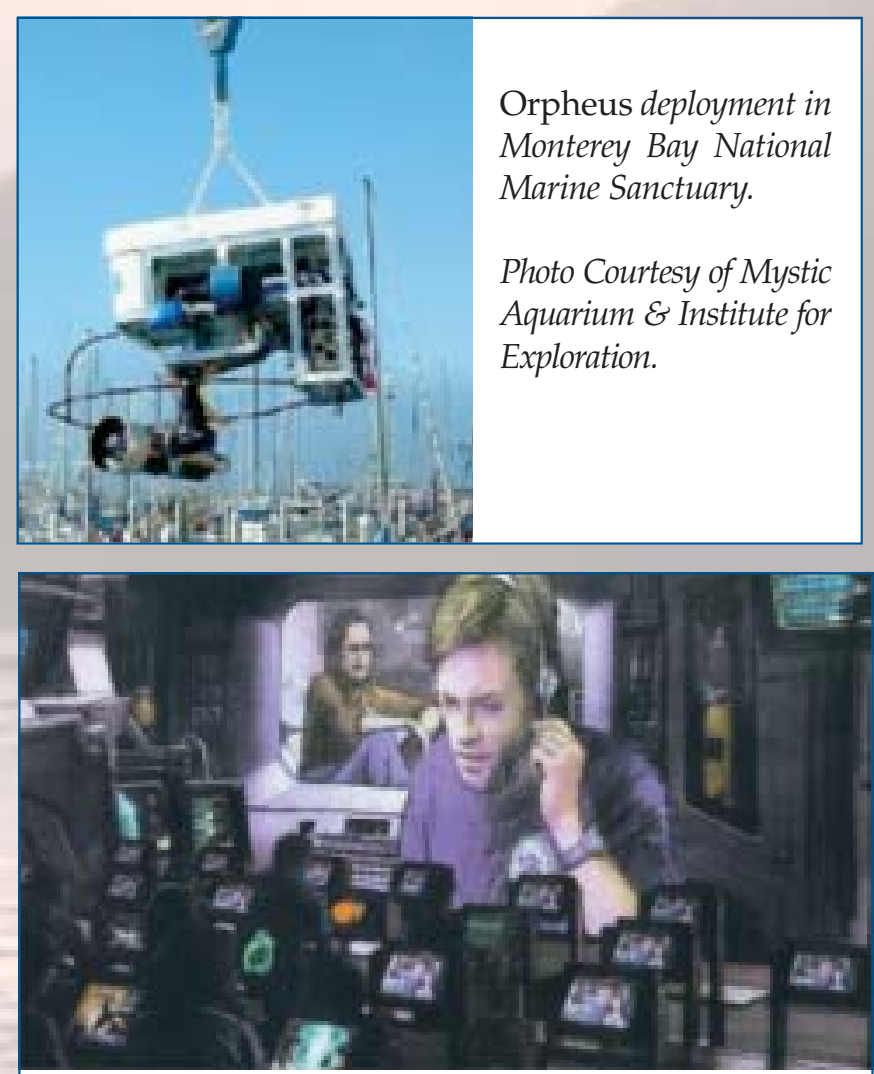

Artist's rendition of audience members at Mystic Aquarium's "Immersion Theater" in Mystic, Connecticut, watch a live expedition from a national marine sanctuary.

Photo Courtesy of Mystic Aquarium \& Institute for Exploration.

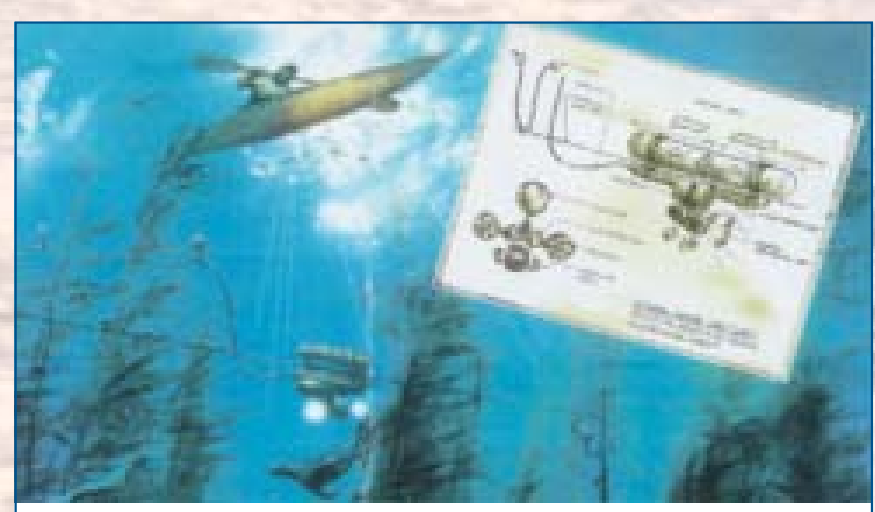

Artist's rendition of Orpheus, deployed in Monterey Bay National Marine Sanctuary.

Photo Courtesy of Mystic Aquarium \& Institute for Exploration.

Photo Courtesy of NOAA 